\title{
A real time capable battery model for electric mobility applications using optimal estimation methods
}

\author{
Jonathan Brembeck Sebastian Wielgos \\ German Aerospace Center (DLR) Oberpfaffenhofen, Institute of Roboticsi and Mechatronics \\ Muenchner Strasse 20, D-82234 Wessling, Germany \\ jonathan.brembeck@dlr.de Sebastian.Wielgos@schaeffler.com
}

\begin{abstract}
In this paper a modeling approach for a Lithium-Ion cell online monitoring and offline benchmarking is proposed. It combines physical modeling in equivalent electric circuit representation with grid tables of cell type characteristic information from laboratory tests. The model is fully parameterized and validated with cells used in the HighVoltage battery pack of DLR research robotic electric vehicle ROboMObil.

Keywords: BMS (BatterieManagementSystem); SOC (StateOfCharge); EKF (ExtendedKalmanFilter); BEV (BatteryElectricVehicle);
\end{abstract}

\section{Introduction}

Nowadays electric mobility gets more and more important. New manufacturing methods give the hope that in the near future vehicles for individual transport can be fully electrified. The use of secondary chemical energy storages is one of the main development tasks in the automotive industry. Besides of the development of new chemical mixtures for higher power density and durability, it is also necessary to develop new embedded systems and advanced algorithms for battery management systems and global energy distribution strategies. The aim of these systems is to give a good estimation for actual and future power availability and health monitoring. This requirement is very complex due to the nonlinear behavior, especially with high performance Lithium-Ion cells. Currently no direct measurement method without destruction of the cell is available to determine the major characteristic parameters and states. In this paper a model for use with recursive online estimation is suggested which gives a good trade-off between modeling accuracy and real-time requirements like low order system or a minimal number of (non)linear subsystems. The proposed algorithm is successfully implemented, parameterized and tested within the ROboMObil project (Figure 1, [Bre11] ), a research platform for future electric mobility developed at the DLR Institute for Robotics and Mechatronics.



Figure 1: ROboMObil test drive

The suggested model is also integrated in the BatteryElectricVehicle model for drive cycle simulations of the overall system in [Eng10]. It is there applied for offline analysis and parameter variation.

\section{Different approaches for cell modeling}

\subsection{Models for offline purposes}

To model the electric behavior of a cell, the obvious thing to do is to use an equivalent circuit. As an example for this type of modeling, the approach from [Böh08] is presented. The cell behavior is separated into three time domains. The impedance 
determines the short-time range while the long-term behavior is incorporated by a voltage source. Finally, the transitional behavior is captured by a number of exponential functions which are overlaid.

Another possibility for cell modeling is based upon impedance spectroscopy measurements. An example for this class of models can be found in [Sti08] with its enhanced equivalent circuit. The level of detail is significantly higher compared to [Böh08] due to the continuous formulation from low to high frequency effects. The following cell characteristics are considered: ohmic resistances, parasitic inductances, charge transfer and double layer capacity, diffusion processes and formation of solid electrical interface.

For purposes of cell design, the Comsol Batteries and Fuel Cells Module ( [Com10] ) is an advanced modeling method. The model is so accurate that it is possible to simulate the concentration of the electrolyte and therefore enables battery engineers to test different combinations of materials and dimensions to optimize the cell behavior.

\subsection{Models for online purposes}

The presented offline models are not applicable to embedded control systems due to their modeling approaches and the relatively complicated representations. Available BatterieManagement Systems, e. g. the widely used system from $I+M E$ Actia, use a predetermined cell characteristic table and a current counting method without considering the transient behavior of the cell.

Another and more advanced approach is the EnhancedSelfCorrecting model by Plett proposed in [Ple04], [Ple04b], [Ple04c] and [Ple04d]. The cell is considered as a system with input "cell current" and output "terminal voltage". The StateOfCharge $l$ is included in the state vector and therefore can be estimated by means of an ExtendedKalmanFilter. The basis of the model is the OpenCircuitVoltage $U_{O C V}$ and the ohmic loss. This is represented in the equivalent circuit in Figure 2.

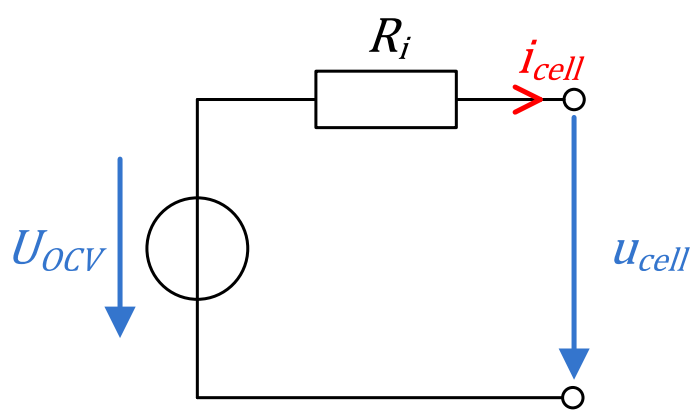

Figure 2: Equivalent circuit representation
Beyond that, this approach takes into account hysteresis effects. The remaining cell dynamics is described by means of a current filter. Therefore, this results in the following output equation:

$$
u_{\text {cell }}=U_{O C V}(l)+h-R_{i} \cdot i_{\text {cell }}+u_{f}
$$

where $h$ is the hysteresis voltage and $u_{f}$ represents the influence of the current filter.

The implementation of the ESC model shows significant optimization potential. There are several better discretization methods than the proposed explicit Euler 1 method. This helps in simulation stability and accuracy for online and offline purpose (see section 3.3 for details). The formulation of the current filter also seems unnecessary complex, It is formulated as an IIR Low Pass Filter as follows: LowPass $=1-$ HighPass,.

Furthermore the computational costly online parameterization of the filter by the use of a dual estimation approach (see [Ple04d] ) can be done offline. In this way the system order can be reduced and therefore the online performance increases. Moreover, the first implementations of the ESC model at DLR have shown problems with the determination of SOC. In addition, the effects of current and temperature on the actual cell capacity are not considered in the ESC model. These can have an enormous influence on the calculation of the SOC, cf. Figure 3.

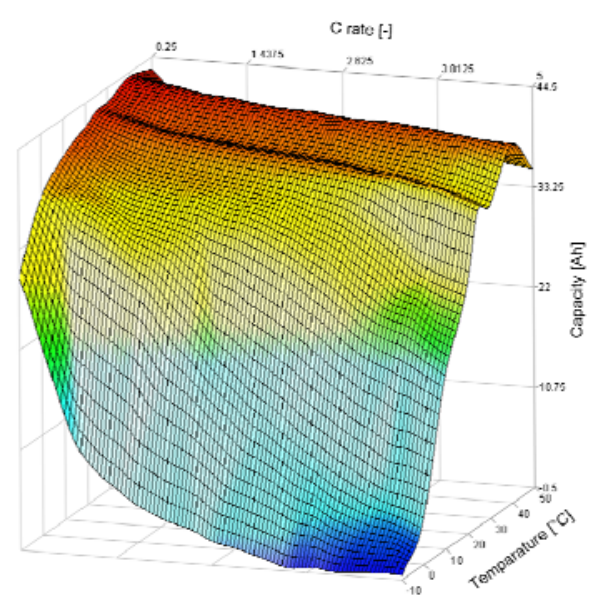

Figure 3: Capacity variation due to temperature and current $\left(C_{\text {Rate }}=C_{N} / I\right)$ 


\subsection{Proposal of the modifiedESC model}

Since we are convinced of the basic approach with the ESC model, we will introduce a modified version that is different in several aspects. In the $m E S C$ approach, the battery cell is represented by a continuous state space model. Through this scheme, the discretization algorithm can be changed and methods with higher accuracy, such as Runge-Kutta 4, can be used. Due to the complex structure and time consuming parameterization of the current filter, it is replaced by a critical damping FIR filter that only contains one parameter. Additionally, the calculation of the $S O C$ is improved by an empirically determined correction factor that accounts for the dependency of temperature and current on the actual cell capacity. Lastly, the model is parameterized using an offline optimization with real training data and then verified by validation data.

\section{3 mESC model details}

\subsection{Derivation and model structure}

The mathematical description of the mESC model is given by the following:

$$
\begin{gathered}
{\left[\begin{array}{c}
l \\
h \\
f_{f}
\end{array}\right]=\left[\begin{array}{c}
-\frac{\eta_{A h} \cdot k_{i}}{C_{N}} \cdot i_{\text {cell }} \\
\left|\frac{\gamma \cdot \eta_{A h} \cdot k_{i}}{C_{N}} \cdot i_{c e l l}\right| \cdot(M-h) \\
-\omega \cdot f_{f 1}+\omega \cdot i_{\text {cell }} \\
\omega \cdot f_{f 1}-\omega \cdot f_{f 2} \\
\omega \cdot f_{f 2}-\omega \cdot f_{f 3} \\
\omega \cdot f_{f 3}-\omega \cdot f_{f 4}
\end{array}\right]} \\
u_{\text {cell }}=U_{O C V}(l)+h-R_{i} \cdot f_{f 4}
\end{gathered}
$$

The differential equation for the SOC $l$ depends on the cell current $i_{\text {cell }}$, the nominal cell capacity $C_{N}$, the Coulombic efficiency $\eta_{A h}$ and the correction factor $k_{i}$. This factor takes into consideration the variation of the cell capacity as shown in Figure 3. Following [Gra02] the correction factor is determined by:

$$
k_{i}=\left\{\begin{array}{c}
c_{i} \cdot i_{\text {cell }}+k_{0} \text { for } \\
\left.i_{\text {cell }}>0 \text { (chrg. }\right) \\
e^{c_{i} \cdot i_{\text {cell }}+\left(k_{0}-1\right) \text { for }} \\
i_{\text {cell }}<0(\text { dchrg. })
\end{array}\right.
$$

$c_{i}$ is a positive constant leading into a straight line for positive cell current, which intersects the ordinate at $k_{0}$. For negative cell current the correction factor is described by an exponential function. The parameters $c_{i}$ and $k_{0}$ are gathered from capacity tests replacing the simple straight line with more accurate look-up tables.

The hysteresis voltage $h$ is described by a more complex equation taking into account the additional factors $M$ (polarization voltage) and $\gamma$ (time constant). The remaining four differential equations describe the optimized fourth order critical damping current filter with the only remaining parameter $\omega$ and its four states $f_{f x}$.

The output equation (3) is similar to the ESC model's output equation but aggregates the influence of the cell current (ohmic loss and current filter) into one summand.

\subsection{Important variables and parameters}

The internal resistance is one of the cell's descriptive variables, which depends on SOC, cell current and temperature resulting in a three dimensional look-up table. This relationship is visualized for room temperature in Figure 4. Considering equation (3) one can imagine easily that the resulting cell voltage varies highly in case of low SOC and high currents due to increase of the internal resistance of the cell. In such cases the cell is in a critical situation and can be damaged irreversible.



Figure 4: Internal cell resistance for $T=25^{\circ} \mathrm{C}$

Another important cell variable is the OpenCircuitVoltage, whose characteristic curve incorporates the relationship between SOC and OCV, as shown in Figure 5. This depiction also shows the hysteresis effects very well. The blue and red curves are measured during charging and discharging of the cell with very low currents respectively. This minimizes excitation of the cell dynamics so that the cell terminal voltage can be considered unloaded. In addition, the influence of the internal resistance is eliminated during the data 
analysis. The polarization voltage is defined as half of the difference between the two curves and therefore also depends on the OCV.

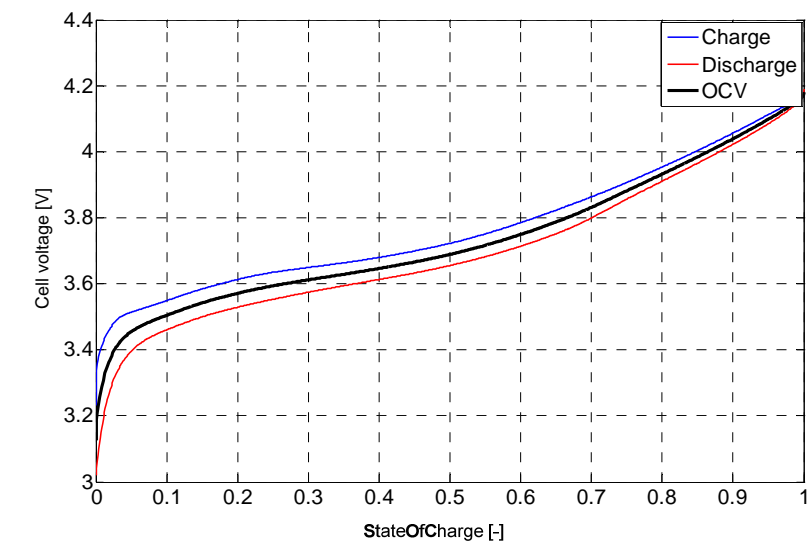

Figure 5: OpenCircuitVoltage

In Figure 6 the polarization voltage is plotted depending on the temperature. It is apparent that temperatures below - have the highest influence.

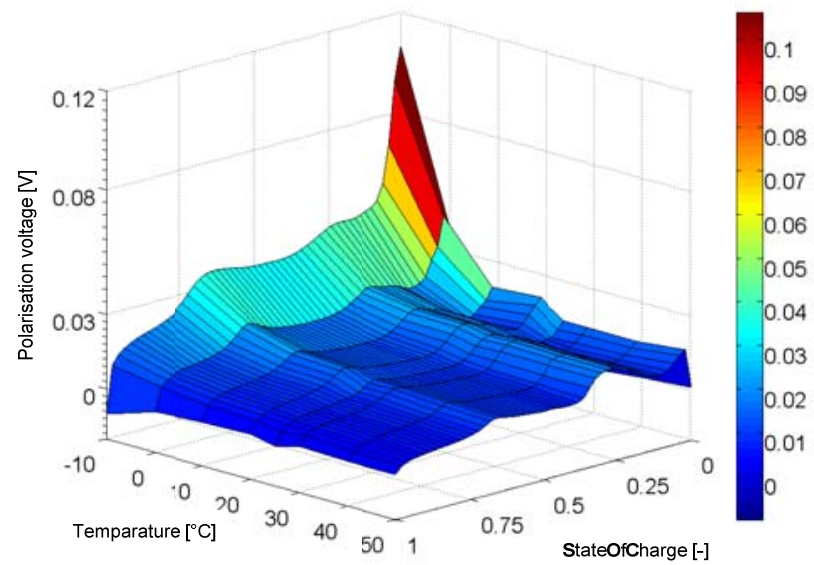

Figure 6: Polarization voltage

Finally, Figure 7 shows the dependency of a exemplary correction factor on at room temperature. Measurement data is collected only for discharge and stored in a look-up table. For charging the data is calculated using equation (4).

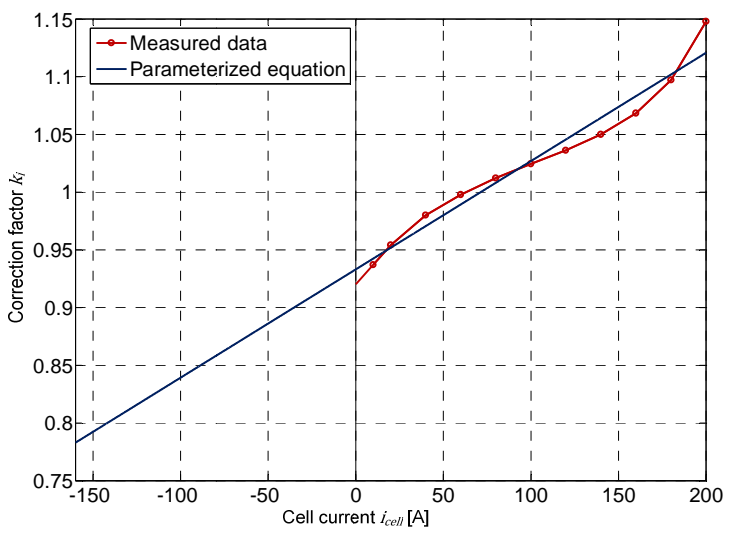

Figure 7: Exemplary identification of $\quad$ for $T=25^{\circ} \mathrm{C}$

The discussed model is implemented in Modelica as part of DLRs ROMOEnergetic library. It is used for offline simulations to optimize the energy management strategies of the vehicle controllers and implemented in the central control unit of the ROboMObil using a rapid prototyping environment. The resulting implementation in DYMOLA is depicted as follows.



Figure 8: mESC model implementation in DYMOLA

\section{3 mESC model and Kalman filtering}

The proposed mESC model has one input, cell current, and one output, cell voltage. These two quantities can be directly measured with high accuracy even in embedded systems. The third quantity is the cell temperatture. It is determined by the use of a thermocouple sensor on the cell surface. This is the same method as applied in the cell test bench (see section 4 for details). To achieve a better estimation performance, a second measurement equation is implemented. The idea in principal is to take constraints into account with a recursive Kalman Filter. For this purpose an additional 
fictitious measurement is introduced. It can be weighted through the tuning of the output covariance matrix. This method is well known as perfect measurements ( [Sim06] chp. 7.5.2). In this way the output equation extends to:

$$
y=\left[\begin{array}{c}
u_{c e l l} \\
l
\end{array}\right]
$$

The first equation is identical to formula (3) and the second one can be derived as follows:

$$
\begin{aligned}
u_{\text {cell }} & \approx U_{\text {OCV }}(l)-R_{i} \cdot i_{\text {cell }} \\
\Rightarrow U_{O C V}(l) & \approx u_{\text {cell }}+R_{i} \cdot i_{\text {cell }} \\
\Rightarrow l \approx l_{\text {meas }} & =U_{O C V}^{-1}\left(u_{\text {cell }}+R_{i} \cdot i_{\text {cell }}\right)
\end{aligned}
$$

The measured SOC is calculated through the inverse OCV $/ U_{O C V}^{-1}$ look-up in combination with a low pass filtering afterwards. This extension allows the ExtendedKalmanFilter to adjust SOC directly and therefore to enforce a physically correct estimation. To use the proposed mESC model in an embedded system for state estimation an EKF is implemented. It handles the nonlinearities through linearization in each time step. In Table 1 the algorithm for discrete systems is shown as found in standard literature (e. g. [Sim06] ).

Table 1: Extended Kalman Filter Algorithm

Initialization

$$
\begin{aligned}
\hat{x}_{0} & =E\left(x_{0}\right) \\
P_{0}^{+} & =E\left[\left(x_{0}-\hat{x}_{0}^{+}\right)\left(x_{0}-\hat{x}_{0}^{+}\right)\right]
\end{aligned}
$$

For $k=1,2, \ldots$

$$
\begin{aligned}
\hat{x}_{k}^{-} & =f_{k-1}\left(\hat{x}_{k-1}^{+}, u_{k-1}\right) \\
P_{k}^{-} & =\Phi_{k-1} P_{k-1}^{+} \Phi_{k-1}^{T}+Q \\
\text { where } \Phi_{k-1} & =\left.\frac{\partial f_{k-1}}{\partial x}\right|_{\hat{x}_{k-1}^{+}} \\
K_{k} & =P_{k}^{-} H_{k}^{T} \cdot\left(H_{k} P_{k}^{-} H_{k}^{T}+R\right)^{-1} \\
\text { where } H_{k} & =\left.\frac{\partial h_{k}}{\partial x}\right|_{\hat{x}_{k}^{-}} \\
\hat{x}_{k}^{+} & =\hat{x}_{k}^{-}+K_{k} \cdot\left(y_{k}-h_{k}\left(\hat{x}_{k}^{-}\right)\right) \\
P_{k}^{+} & =\left(I-K_{K} \cdot H_{k}\right) \cdot P_{k}^{-}
\end{aligned}
$$

Through the fact that our estimation model (equations (2) and (5)) is in a continuous state space formulation, it is necessary to discretize the model in each time step. This can be done by several methods. The easiest way is by the use of an Euler1 method. Because of the poor stability (all poles have to be placed within the unit circle of the time dependent complex pane) we suggest using a Trapezoid method (eq. (7)). It guarantees that the prediction step $\left(\hat{x}_{k}^{-}\right)$ is always stable, even for a large sampling time $T_{s}$, as long as all poles of the continuous system stay in the left half of the complex pane.

$$
\frac{\dot{x}\left(t_{k}\right)+\dot{x}\left(t_{k}+T_{s}\right)}{2}=\frac{x\left(t_{k}+T_{s}\right)-x\left(t_{k}\right)}{T_{s}}
$$

After some calculations the prediction step is described as follows,

$$
\hat{x}_{k}^{-}=\hat{x}_{k-1}^{+}+\left(I-\frac{T_{s}}{2} F_{k-1}\right)^{-1} \cdot T_{s} f_{k}
$$

where $F_{k-1}$ is the Jacobi matrix of the continuous system with state vector $k-1$. To receive the transition matrix $\Phi_{k-1}$ we have to solve the following equation:

$$
\Phi_{k-1}=\left(I-\frac{T_{s}}{2} F_{k-1}\right)^{-1} \cdot\left(I+\frac{T_{s}}{2} F_{k-1}\right)
$$

For reason of numerical stability all matrix inversions are done by solving a linear equation problem of the form $A \cdot x=b$. This can be done with LU_solve2 from ModelicaStandard. In conclusion we have an Extended Kalman Filter algorithm which has $O\left(n x^{3}\right)$-flops more than the original filter, but outperforms it due to the fact that the sampling time can be chosen much larger. This is only limited by Shannon's sample theorem for sampling the measured signals in real-time. Another limit is that the linearization of the EKF causes a mean and covariance propagation which is only valid to the first order. This is due to Taylor series expansion being truncated after the first term. The second issue can be improved by the use of higher order methods like the Unscented Kalman Filter, [Mer04] .

\section{Parameterization and validation}

In the ROboMObil project we were able to obtain a high performance cell from Li-Tec industries. It has a nominal capacity of 40 Ah and with its security features it is fully capable for series production. All cell measurement for parameterization, testing, and validations were done with a Vötsch VT4011 environment simulator and a BaSyTec battery testing system. The model parameter optimization is accomplished with MOPS (see [Joo08] for more information) on the Linux cluster of DLR RM Institute. The detailed procedure and the necessary test cycles are explained in [Wie10]. The list of tuned parameters is given in Table 2 . 
Table 2: List of optimized parameters

$\gamma \quad \begin{aligned} & \text { Time constant for rate of change of the } \\ & \text { hysteresis voltage }\end{aligned}$
$f_{g} \quad \begin{aligned} & \text { Cut-off frequency of the fourth order } \\ & \text { current filter with critical damping }\end{aligned}$
$f_{l} \quad \begin{aligned} & \text { Cut-off frequency of the first order low } \\ & \text { pass filter for smoothing the "measured" }\end{aligned}$
$\begin{aligned} & \text { SOC needed due to the extended output } \\ & \text { equation }\end{aligned}$
$Q, R \quad \begin{aligned} & \text { Covariance matrices for optimal EKF } \\ & \text { settings (weighting of prediction by } \\ & \text { means of the model and correction due to } \\ & \text { the measured values) }\end{aligned}$

The optimization process can be summarized as shown in Figure 9. As quality criterions cell voltage and SOC are compared to their respective references using the so-called fit value. The fit value weights the reference to the simulated characteristic vector and can be calculated as follows:

$$
\text { Fit }=\left(1-\frac{\sqrt{\sum_{i=1}^{n}\left(\left|y_{h ; i}-y_{i}\right|^{2}\right)}}{\sqrt{\sum_{i=1}^{n}\left(\left|y_{i}-\frac{1}{n} \cdot \sum_{i=1}^{n} y_{i}\right|^{2}\right)}}\right) \cdot 100
$$

Since a direct comparison of SOC is not possible, the actually and effectively moved amount of charge at the terminals of the cell is used.



Figure 9: MOPS optimization process

For the validation of the estimated model parameters a testbench experiment by means of a simulated drive cycle is used. The observer scheme is shown in Figure 10. The aim is to produce an accurate estimate of the battery StateOfCharge, which is a decisive input for the function of the energy management strategy. In this observer, the input $u$ of the battery model is the measured current, while the model output $y_{m}$ is the voltage of a single cell.

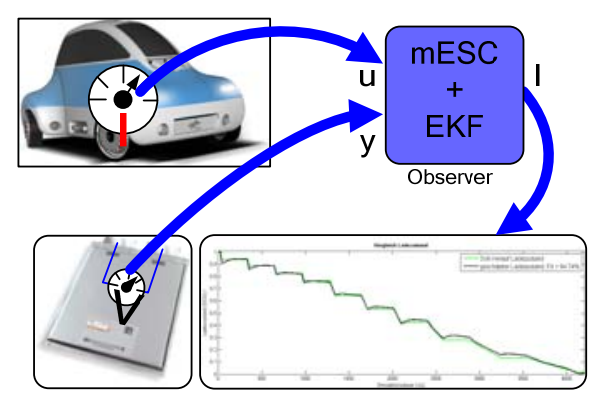

Figure 10: mESC observer structure

The model used in the observer was parameterized by an offline optimization that uses measurements test cycles and the characteristics of a single cell as training data. In order to validate the resulting parameterization, the battery was connected to an electrical power supply/load and tested with a simulated drive cycle. For this simulation, a model of the longitudinal dynamics of the ROboMObil was developed in Modelica which calculates the energy flow of all the power consumers in the electrical system [Eng10] .


Figure 11: Artemis Road velocity profile and power consumption 
A closed-loop controller calculates the actuator demands in order to follow the specified driving cycle. In this way, the required electrical power is calculated. Figure 12 shows a normalized Artemis Road cycle, which is synthesized by stochastic calculations from real recorded driving data [And04] - This reflects real driving behavior significantly better than the purely synthetic ECE15 driving cycle used in the homologation of European vehicles. The calculated electrical power flow is then taken from a Dymola simulation and scaled to one battery cell. This data is used on the HIL test bench to give the Lithium-Ion cell the appropriate load current. The results of this test, including the current, voltage, as well as the cell temperature are used to test the mESC model with EKF in the offline simulation. The test results are shown in Figure 12, where the measured and estimated values are compared to each other. Evidently, the voltage values agree very well. With almost 93\% accuracy, the accordance of the effective charge amount delivers a good result and shows that the selected approach with the modelbased observer is of practical use.

\section{Conclusions and future work}

We have introduced a modeling approach for Lithium-Ion cells that shows good performance and was implemented within a practical application (ROboMObil). Future development will extend the model with real-time capable temperature dynamics as suggested in [Che09], [Mi07] and [Mat08] . This should lead to better results in prediction of power availability during critical situations like sub-zero temperatures. Furthermore validation tests on the rapid prototyping embedded systems in the ROboMObil are planned. In the first weeks of
January 2011 we are able to accomplish roller bench experiments with the ROboMObil. We are looking forward to gaining new insights from the recorded measurement data of the energy system. The results will be presented in an upcoming publication.

\section{Acknowledgment}

We would like to thank Prof. M. Otter for his support regarding implementation. His extensive knowledge of robust and reliable numerical matrix calculus was instrumental in achieving these good results. Furthermore we would like to thank LionSmart GmbH for the open discussions in points of model implementation.

\section{References}

[And04] André, M. (2004). The ARTEMIS European driving cycles for measuring car pollutant emissions. Science of The Total Environment , 334-335, 73-84.

[Com10] Batteries and Fuel Cells Module. Retrieved 2010, from http://www.comsol.com/showroom/gallery/686/

[Böh08] Böhm, K. A. (2008). Charakterisierung und Modellierung von elektrischen Energiespeichern für das Kfz. Aachen: Shaker Verlag.

[Bre11] Brembeck, J., Ho, L. M., Schaub, A., Satzger, C., \& Hirzinger, G. (2011). ROMO - the robotic electric vehicle - Submitted for publication. IAVSD

[Eng10] Engst, C., Brembeck, J., Otter, M., \& Kennel, R. (2010). Object-Oriented Modelling and RealTime Simulation of an Electric Vehicle in Modelica. Technische Universität München


Figure 12: Experiment results from Artemis Road Drive Cycle Test 
[Che09] Cheng, L., Ke, C., Fengchun, S., Peng, T., \& Hongwei, Z. (2009). Research on thermophysical properties identification and thermal analysis of EV Li-ion battery., Vehicle Power and Propulsion Conference, 2009. VPPC '09. IEEE, 2009, $1643-1648$

[Gra02]Graaf, R. (2010). Simulation hybrider Antriebskonzepte mit Kurzzeitspeicher für Kraftfahrzeuge. Ika RWTH, Aachen.

[iME10]i+ME ACTIA. (n.d.). Retrieved 11 2010, from http://www.ime-actia.com/download/ IR11652_BMS_GB.pdf

[Joo08] Joos, H.-D., Bals, J., Looye, G., Schnepper, K., \& Varga, A. (2008). MOPS: Eine integrierte optimierungsbasierte Entwurfsumgebung für mehrzielige, parametrische Analyse und Synthese. DGLR Workshop Systemidentifizierug, Parameterschätzung und Optimierung.

[Lio10] LionSmart. (2010). Retrieved 11 2010, from http://www.lionsmart.com/

[Mat08] Matsushita, T., Yabuta, K., Tsujikawa, T., Matsushima, T., Arakawa, M., \& Kurita, K. (2008). Construction of three-dimensional thermal simulation model of lithium-ion secondary battery., Telecommunications Energy Conference, 2008. INTELEC 2008. IEEE 30th International, 2008, 1 -6

[Mer04] Merwe, R. , Wan, E., \& Julier, S. (2004). SigmaPoint Kalman Filters for Nonlinear Estimation and Sensor-Fusion: Applications to Integrated Navigation, AIAA Guidance, Navigation, and Control Conference and Exhibit, 2004

[Mi07] Mi, C., Li, B., Buck, D., \& Ota, N. (2007). Advanced Electro-Thermal Modeling of Lithium-Ion Battery System for Hybrid Electric Vehicle Applications., Vehicle Power and Propulsion Conference, 2007. VPPC 2007. IEEE, 2007, 107 -111

[Ple04] Plett, G. (2004). High-performance batterypack power estimation using a dynamic cell model. Vehicular Technology, IEEE Transactions on , 53 (5), 1586-1593.

[Ple04b]Plett, G. L. (2004). Extended Kalman filtering for battery management systems of LiPBbased HEV battery packs: Part 1. Background. Journal of Power Sources , 134 (2), 252-261.

[Ple04c] Plett, G. L. (2004). Extended Kalman filtering for battery management systems of LiPBbased HEV battery packs: Part 2. Modeling and identification. Journal of Power Sources , 134 (2), 262-276.

[Ple04d]Plett, G. L. (2004). Extended Kalman filtering for battery management systems of LiPBbased HEV battery packs: Part 3 . State and parameter estimation. Journal of Power Sources , 134 (2), 277-292.
[Sim06] Simon, D. (2006). Optimal State Estimation: Kalman, $\mathbf{H}$ Infinity, and Nonlinear Approaches (1. Auflage ed.). Wiley \& Sons.

[Sti08] Stiftl, J. (2008). Modellierung und Bewertung von Fahrzeugen mit seriellem Plug-In Hybridantrieb. Hochschule Karlsruhe Technik und Wirtschaft: Diplomarbeit.

[Wie10] Wielgos, S., Brembeck, J., Otter, M., \& Kennel, R. (2010). Development of an Energy Management System for Electric Vehicles Design and System Simulation. Technische Universität München. 